\title{
Diseño e innovación radical desde una visión sistémica: el caso de las pymes industriales de Guayaquil-Ecuador
}

\begin{abstract}
Resumen
Las investigaciones sobre diseño e innovación radical continúan contribuyendo al surgimiento de metodologías aplicables principalmente en contextos europeos, dado su origen. Por ello, nuestro objetivo fue estudiar la relación diseño e innovación radical, desde una visión sistémica, centrada en el proceso de innovación en pymes industriales de la ciudad de Guayaquil. Se optó por una investigación exploratoria y descriptiva, bajo una mirada pragmática que combina herramientas cualitativas y cuantitativas, lo que permitió tener una visión más integral y completa del fenómeno. La metodología contempló dos fases una documental y otra empírica que consistió en una entrevista a profundidad a 66 pymes industriales. Entre las principales conclusiones destacamos que, el diseño se integra al proceso de innovación radical, cuando los diseñadores tienen habilidades estratégicas, cuando la gerencia posee una cultura hacia el uso del diseño y esté identificado con el potencial del diseño y cuando existen políticas públicas que ponen en valor al diseño en la región. Es decir, no basta con tener diseñadores especializados, se requiere abordar no solo el desempeño individual de las partes, sino también sus interacciones, donde confluyen tres componentes: i. El Sistema nacional de innovación (SNI), puesto que este ejercerá muchas condicionantes al desarrollo de la innovación en un territorio en particular; ii. El sistema de gestión de innovación propio de la empresa (SGI), de vital importancia para la fluidez de la innovación y iii. El accionar del diseño como actor focal en esta red de interacciones.
\end{abstract}

\author{
Ruth Matovelle \\ Doctora en Diseño \\ Escuela Superior Politécnica \\ del Litoral, Guayaquil, Ecuador \\ Correo electrónico: \\ rmatovel@espol.edu.ec \\ ๑ orcid.org/0000-0001-8909-8988 \\ Google Scholar \\ Manuel Lecuona \\ Doctor en BBAA \\ Universidad Politécnica de València, \\ Valencia, España. \\ Correo electrónico: \\ mlecuona@igd.upv.es \\ ๑ orcid.org/0000-0002-9642-9738 \\ Google Scholar

\begin{abstract}
Bernabé Hernandis
Doctor en Diseño

Universidad Politécnica de València. Valencia, España.

Correo electrónico: bhernand@upv.es ๑ orcid.org/0000-0002-5697-6382

Google Scholar
\end{abstract}

Recibido: septiembre 11 de 2019 Aprobado: octubre 22 de 2020

Palabras clave:

diseño, innovación radical, sistémico. 


\section{Design and radical innovation from a systemic perspective: the case of industrial Small and Medium-sized Enterprises (SMEs) in Guayaquil-Ecuador}

\begin{abstract}
Research on design and radical innovation continues to contribute to the emergence of methodologies applicable mainly in European contexts, given their origin. Therefore, our objective was to study the relationship between design and radical innovation from a systemic perspective, focused on the innovation process in industrial SMEs in the city of Guayaquil. An exploratory and descriptive research was chosen under a pragmatic perspective that combines qualitative and quantitative tools, which allowed to have a more comprehensive and complete vision of the phenomenon. The methodology included two phases, a documentary and an empirical one, which consisted of an in-depth interview with 66 industrial SMEs. Among the main conclusions, we highlight that design is integrated into the radical innovation process when designers have strategic skills, when management has a culture towards the use of design and is identified with the potential of design, and when there are policies that put in value to design in the region. That is, it is not enough to have specialized designers, it is necessary to address not only the individual performance of the parts but also their interactions where three components converge: i. The National Innovation System (SNI), since it will exert many conditions to the development of innovation in a particular territory; ii. The own innovation manag ement system (IMS) of the company which is of vital importance for the fluidity of innovation; and iii. The action of design as a focal actor in this network of interactions.
\end{abstract}

Key words:

design, radical innovation, systemic. 


\section{Introducción}

Las innovaciones radicales son transformaciones importantes de productos y servicios existentes, y requieren de un enfoque disciplinado para identificar y ejecutar rigurosamente las mejores ideas, que implican cambios al interior y exterior de la organización (Chandy \& Tellis, 2000; Jones \& Samalionis, 2008). La capacidad de desarrollar y comercializar con éxito innovaciones radicales se constituye en una "capacidad dinámica", difícil de imitar. Estas capacidades dinámicas permiten a los gerentes adaptar, integrar y desplegar habilidades organizacionales internas y externas para lograr la alineación con el entorno empresarial cambiante (Slater, Mohr \& Sengupta, 2014). Una percepción común en el campo de la innovación es que estas capacidades dinámicas de las empresas dejan por fuera a las grandes empresas, las cuales rara vez introducen innovaciones radicales de productos, por su inercia y burocracia. Como resultado, las innovaciones radicales tienden a provenir de pequeñas empresas, tesis identificada como la "maldición del titular", comúnmente aceptada en los relatos académicos y populares de la innovación radical, donde se evidencian la caída de grandes empresas mientras se catapulta las pequeñas empresas en el liderazgo del mercado (Damanpour, 1991; Rothwell,1994; Chandy \& Tellis, 2000; Leifer et al., 2000; Tether, 2005).

Ahora bien, desde la óptica disciplinar del diseño, se ha generalizado que este es un instrumento de innovación. El diseño se refleja de manera global en la innovación. Su modo de pensamiento holístico es útil para la liberalización entre las funciones y las culturas, transforma la materia prima en productos que la gente puede usar realmente, o inclusive anticiparse a las necesidades futuras de un mercado; constituyéndose así en una valiosa aportación a la innovación radical (Borja de Mozota, 2006; Cotec, 2009; Cruickshank, 2010; Hobday et al., 2011; Norman \& Verganti, 2014). Los resultados de investigaciones empíricas han evidenciado que el acercamiento entre la empresa y los 
proyectos innovadores le confiere al diseño un valor estratégico, lo que detona el desarrollo de nuevas habilidades y roles del diseño para con el proceso de innovación (Noble, 2011). Dado este contexto, nace este proyecto de investigación como componente de una tesis doctoral, cuyo objetivo es analizar al diseño y su relación con la innovación radical bajo una mirada sistémica, y así identificar la caracterización del fenómeno en empresas ecuatorianas.

Ante ello, el primer reto de esta investigación fue identificar el tipo de empresa en el que podría existir el proceso de innovación radical. Respecto al tamaño de empresa, se seleccionaron las pymes por su dinamismo en la ejecución de innovación radical — tal como lo explicara Damanpour (1991) en líneas anteriores - y también porque tienen un rol protagonista en el económica ecuatoriana —dado que al 2015 aportaron más del 25\% del PIB no petrolero del país, con el 90\% de las unidades productivas, y su generación de mano de obra bordea el 70\% de toda la población económica activa (INEC, 2015; Carranco, 2017)_. Para su clasificación se empleó el Código Orgánico de la Producción, Comercio e Inversiones, esto es: por el número de empleados, el valor bruto de las ventas anuales y el valor de los activos totales (ver Tabla 1).

Tabla 1. Clasificación de empresas por Tamaño

\begin{tabular}{lll}
\hline Clasificación de las empresas & Volúmenes de ventas anuales & Personal ocupado \\
\hline Microempresa & Menor a 100.000 & 1 a 9 \\
\hline Pequeña empresa & De 100.001 a 1'000.000 & 10 a 49 \\
\hline Mediana empresa "A" & De 1'000.001 a 2'000.000 & 50 a 99 \\
\hline Mediana empresa "B" & De 2'000.001 a 5’000.000 & 100 a 199 \\
\hline Grande empresa & De 5'000.001 en adelante & 200 en adelante \\
\hline
\end{tabular}

Fuente: INEC, 2015. 
Respecto al sector de la economía, el Servicio de Rentas Internas afirmó en 2017 que las ventas del sector industrial de elaboración de alimentos es la de mayor relevancia (33\%), le siguen, en orden de importancia, productos y sustancias químicas (9\%) y Bebidas (6,0\%) (Cámara de Industrias de Guayaquil, 2019). Como elemento disruptivo, se encuentra la regulación que sufrió el sector alimenticio impuesta por el gobierno nacional (2015), que consistió en la implementación de un sistema de etiquetado nutricional tipo semáforo a los alimentos procesados, su objetivo es el de informar a los consumidores la cantidad de sal, azúcar y grasa en los productos alimenticios. Adicional a esto, Emma Peterson, analista de investigación del perfil de hábitos de consumo de los latinoamericanos, afirma que América Latina ha empezado a experimentar dos fenómenos determinantes: el estrechamiento de las familias y la necesidad de consumir productos más sanos con un sistema de producción sostenible (Cárdenas, 2015). Todo esto revolucionó la industria alimenticia en el Ecuador, hacia un campo disruptivo por supervivencia, y origino la oportunidad de esta investigación, para conocer los detalles del proceso de innovación y su relación con el área de diseño.

\section{Método}

Esta investigación es de tipo exploratoria y descriptiva, desarrollada bajo una mirada pragmática que combina herramientas cualitativas y cuantitativas. La metodología contempló dos fases: la documental y la empírica.

\section{Fase documental}

Corresponde a un estudio exploratorio que tiene como idea principal obtener un conocimiento más amplio respecto al tema de estudio y, por lo general, forma parte introductoria de un estudio descriptivo; se realiza con el objetivo de "preparar el terreno" para el desarrollo de los otros tipos de investigación 
(Namakforoosh, 2005). Para ello, se emplearon las técnicas de análisis de contenido y consulta a expertos a fin de determinar un marco de referencia; se realizó un análisis conceptual y revisión de la literatura para situar en contexto el tema tratado e identificar las variables en común entre distintos autores con miras al entendimiento y clarificación de los conceptos básicos y centrales que, desde la óptica del diseño, contribuyen a la generación de innovación radical. La consulta a 15 expertos en el tema de diseño e innovación se implementó como contraste de los resultados del análisis anterior y permitió ajustar el planteamiento conceptual y determinar las variables del estudio.

\section{Fase empírica}

Con el propósito de comprender e interpretar el objeto de estudio, con una lógica inductiva, a través de las percepciones y significados producidos por las experiencias de 66 gerentes responsables de proyectos de innovación en pymes industriales de la ciudad de Guayaquil, se les consultó su opinión sobre las características del diseño en el proceso de innovación radical. Dada la inexistencia de estudios similares en el territorio ecuatoriano o en la región de América Latina y el Caribe, se procedió con el diseño de un modelo propio de encuesta. Para ello, ser utilizó como marco referencial el Manual de Oslo, específicamente las novedades de su tercera edición, para medir la dimensión sistémica de la innovación y las características específicas de las economías en desarrollo, aplicable al caso de Ecuador (OCDE \& EUROSTAT, 2005). Se consideró también la teoría de Zurlo \& Cautela (2014), quienes identifican factores que estimulan y promueven el proceso de diseño en el desarrollo de la innovación: nivel de uso, fuentes creativas, herramientas de conocimiento del diseño y capacidades básicas del diseñador. Finalmente, el cuestionario se construyó con un patrón de preguntas abiertas y cerradas orientadas a identificar todo el proceso de innovación, incluyendo la aportación de comentarios espontáneos. Lo conforman cinco bloques: información general, 
innovaciones logradas, gestión de la innovación radical, interacción diseño e innovación radical y personal del área de diseño.

La muestra partió de una población de 165 pymes, que incluye empresas pequeñas y medianas $A$ y $B$; se obtuvo una muestra aleatoria estratificada resultado de separar los elementos de la población en grupos que no se superponen, Ilamados estratos; se seleccionó una muestra aleatoria simple de cada estrato, por lo que la muestra estaría conformada por 66 empresas (36 pequeñas,12 medianas A y 18 medianas B). Se usó el criterio conservador pero válido de asumir que la proporción dentro de cada estrato era de 0.5, pues este valor para la proporción es el que maximiza la varianza, se definió el error máximo permitido como 0.04 o 4\% y un nivel de confianza de 95\%. Para el tratamiento y análisis de datos se usó el software SPSS V20 y se obtuvo la media aritmética y la desviación estándar. Se aplicó el cuestionario, mediante entrevista personal, en el segundo semestre del 2018.

\section{Resultados}

A partir de los hallazgos encontrados en la fase documental se obtuvo la interpretación sistémica del fenómeno diseño e innovación radical, estos hallazgos corresponden a los resultados de procesos empíricos ejecutados en empresas europeas, los cuales identifican la interacción de tres componentes: 1. El Sistema nacional de innovación (SNI) del territorio, puesto que este ejercerá muchas condicionantes al desarrollo de la innovación en forma particular; 2. La gestión innovadora de la empresa, y 3. El accionar del diseño como actor focal en esta red de interacciones, los que en conjunto conformarían el sistema diseño-innovación radical (Yannou et al. 2011). El componente anterior se alimenta de conocimiento proveniente de diversas fuentes internas y externas a la empresa, donde la conexión a los modelos socioculturales, actuales y futuros, y el imaginario de nuevos significados, 
son de vital importancia en su desarrollo (Chesbrough, 2003; 2006; Battistella et al., 2012).

Además de lo anterior, la fase empírica realizada en las pymes industriales del sector alimenticio de la ciudad de Guayaquil basó su observación teniendo en cuenta la visión sistémica del fenómeno, lo que permitió la extracción de los rasgos característicos del territorio.

\section{Componente 1. El Sistema nacional de innovación (SNI)}

Se encontraron numerosos estudios realizados por Lemarchand, G. a. (2010), Botella, C., \& Suárez, I. (2012), CEPAL (2013) y BID (2016), que han dedicado sus esfuerzos y recursos en medir los Sistemas Nacionales de Innovación de la región de América Latina, y en forma particular el de Ecuador. Esta información sirvió de base para realizar una visión actualizada del SNI del Ecuador al 2017. Dada su extensión, en este artículo solo se incluye información de 5/19 variables sugeridas por Zúñiga (2010).

1. Gastos de I+D. Ecuador invierte en I+D apenas el 0,44\% del PBI, mientras que en la región de América Latina y el Caribe el 0.75\%, lo que denota una brecha considerable a nivel regional (INEC, 2015).

2. Políticas Públicas de Ciencia, tecnología e Innovación (CTI). De acuerdo con la Red de Indicadores en Ciencia y Tecnología Iberoamericana e Interamericana (RICYT), Ecuador tiene un total de 27 instrumentos habilitados para el respaldo de las políticas de CTI al 2017 (ver Figura 1). El $48 \%$ de los instrumentos corresponde al fomento de recursos humanos. Un $0 \%$ tienen los ejes de infraestructura y áreas estrategias, los que implican: fomentar la creación y mejora de equipamiento e infraestructura; fondos 
para la creación de clúster, polos tecnológicos e incubadoras de empresas; programas de áreas prioritarias; y fondos sectoriales (RICYT, 2017).

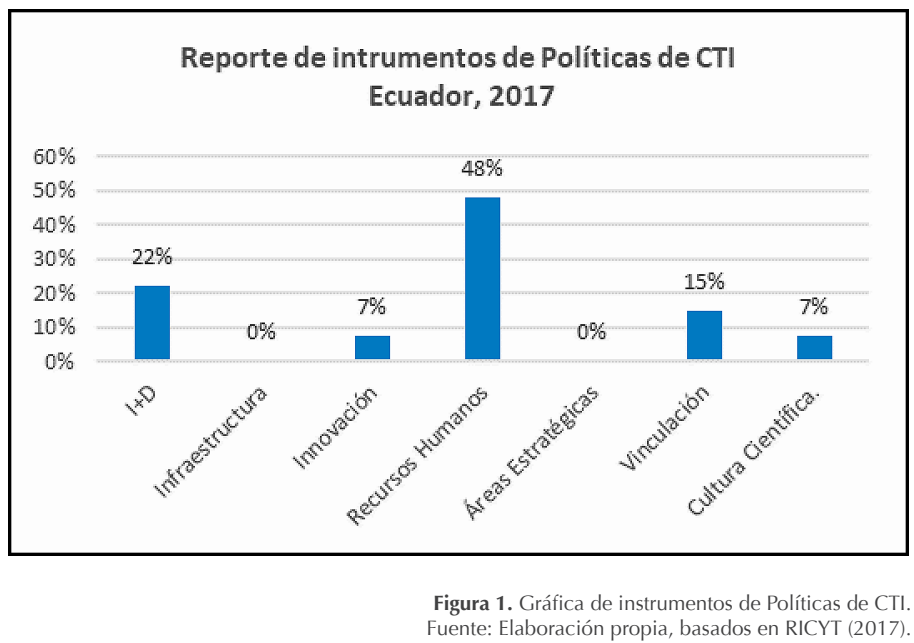

El eje especifico de Innovación cuenta con tan solo dos instrumentos para impulsar esta política, no existe ningún programa que promueva la comprensión del impacto del diseño en la innovación que permita comprender a las empresas el rol protagonista del diseño como motor de innovación.

3. Investigadores. La cantidad de investigadores representan una tasa de 1,59\% por cada 1.000 personas de la Población Económicamente Activa (PEA), superando a la tasa promedio de América Latina que llega a 1,30\%, al 2014. En términos de publicaciones por habitantes, Ecuador está significativamente por debajo del promedio de la región. Para 2010 ALC alcanzó 11,20 publicaciones por cada 100.000 habitantes (Guaipatín \& Schwartz, 2014). 
4. Protección de la propiedad intelectual. El Índice Internacional de Propiedad Intelectual (IPRI) al 2017 ubica al Ecuador, en la posición de 93/127; tiene los niveles más bajos que todos los países de referencia de la región de América Latina y el Caribe (Property Rights Alliance, 2017).

5. Clima de Negocios. Para que una empresa innove, debe desarrollarse en un clima de negocios razonable, sin excesiva regulación gubernamental y con un entorno jurídico y competitivo que favorezca la inversión y los negocios (Guaipatín \& Schwartz, 2014). El Report Doing Business, que mide el clima de negocios a nivel mundial, situó al Ecuador en el puesto 114/190 en el año 2017, y al compararlo con países de la misma región (Colombia 53/190, Perú 54/190) evidencia que existe mucho por hacer con respecto a clima de negocios. En Ecuador es más difícil empezar nuevos negocios, en términos de días y del número de procedimientos requeridos para su registro, así como también debido al alto costo de estos procedimientos y el monto que el empresario debe tener disponible antes de registrar la empresa (Doing Business, 2017).

\section{Componente 2. La Gestión innovadora de la empresa}

La consulta a la empresa permitió identificar sus particularidades como: tamaño, mercado, años de actividad empresarial, innovaciones logradas, tipo de innovaciones, nivel de novedad de la innovación, actividades realizadas para introducir la innovación, monto invertido, fuentes de información, medios de financiamiento, los objetivos que dieron origen a la innovación, los factores de éxito, estructura organizacional y equipos de trabajo. El análisis estadístico descriptivo de frecuencias y el análisis de Chi-cuadrada de estas variables, evidenció las características de este componente: 
1. El tamaño de la empresa es el determinante más consistente de la innovación, tiene un efecto positivo sobre la probabilidad de innovar en productos y en procesos en los países en desarrollo.

2. Antigüedad de las Empresas. Existe una consideración de una relación negativa entre la antigüedad de la empresa y la innovación radical, lo que implica que las empresas son cada vez más incapaces de generar nuevas o importantes innovaciones a medida que envejecen. Esta afirmación se comprobó por medio del índice estadístico Chi-square "Chi-cuadrada", el que realiza una prueba de independencia de variables (Castañeda et al., 2010), se analizó si la variable "tipo de innovación", guarda alguna relación con la variable "años de actividad".

La Tabla 2 muestra la tabulación de las frecuencias. Las empresas con menos de 29 años de actividad realizaron 35 innovaciones de "producto nuevo", las empresas mayores de 30 años realizaron 9; con respecto a "productos con cambios significativos" las de menos de 29 años hicieron 7, versus 15 las mayores de 30 años. 
Tabla 2. Tabulación variables años de antigüedad y tipo de innovación

\begin{tabular}{|c|c|c|c|c|}
\hline & & \multicolumn{2}{|c|}{ ¿Cuál innovación } & \multirow[b]{2}{*}{ Total } \\
\hline & & Producto Nuevo & $\begin{array}{c}\text { Producto con } \\
\text { cambios significativos }\end{array}$ & \\
\hline \multirow{16}{*}{$\begin{array}{l}\text { Años de } \\
\text { actividad de la } \\
\text { empresa }\end{array}$} & \multirow{2}{*}{0 a 4 años } & 9 & 0 & 9 \\
\hline & & $13,6 \%$ & $0,0 \%$ & $13,6 \%$ \\
\hline & \multirow{2}{*}{5 a 9 años } & 7 & 1 & 8 \\
\hline & & $10,6 \%$ & $1,5 \%$ & $12,1 \%$ \\
\hline & \multirow{2}{*}{10 a 19 años } & 10 & 3 & 13 \\
\hline & & $15,2 \%$ & $4,5 \%$ & $19,7 \%$ \\
\hline & \multirow{2}{*}{20 a 29 años } & 9 & 3 & 12 \\
\hline & & $13,6 \%$ & $4,5 \%$ & $18,2 \%$ \\
\hline & \multirow{2}{*}{30 a 39 años } & 4 & 6 & 10 \\
\hline & & $6,1 \%$ & $9,1 \%$ & $15,2 \%$ \\
\hline & \multirow{2}{*}{40 a 49 años } & 2 & 3 & 5 \\
\hline & & $3,0 \%$ & $4,5 \%$ & $7,6 \%$ \\
\hline & \multirow{2}{*}{50 a 59 años } & 2 & 4 & 6 \\
\hline & & $3,0 \%$ & $6,1 \%$ & $9,1 \%$ \\
\hline & \multirow{2}{*}{60 años o más } & 1 & 2 & 3 \\
\hline & & $1,5 \%$ & $3,0 \%$ & $4,5 \%$ \\
\hline \multirow{2}{*}{ Total } & & 44 & 22 & 66 \\
\hline & & $66,7 \%$ & $33,3 \%$ & $100,0 \%$ \\
\hline
\end{tabular}

Fuente: elaboración propia.

La Tabla 3 muestra el resultado del análisis estadístico Chi-cuadrada, el índice más importante es el de la columna 3 (Sig. asintótica) que en estadística se refiere como el valor $p$. En general un valor $p$ menor de .05 se 
considera significativo y un valor mayor de .05 no significativo (Castañeda et al., 2010). En este caso el valor es menor a .05 (.022), con lo cual se concluye que la prueba Chi cuadrada fue significativa y, por lo tanto, las variables sí son dependientes.

Tabla 3. Prueba Chi-square de independencia de variables

\begin{tabular}{lccc}
\hline & Valor & gl & Sig. asintótica (2 caras) \\
\hline Chi-cuadrado de Pearson & $16,353 \mathrm{a}$ & 7 &, 022 \\
\hline Razón de verosimilitud & 18,803 & 7 &, 009 \\
\hline Asociación lineal por lineal & 14,477 & 1 &, 000 \\
\hline N de casos válidos & 66 & & \\
\hline Fuente: elaboración propia. & &
\end{tabular}

Esta prueba se aplicó con el objetivo de evidenciar, en esta localidad, la tesis de la "maldición del titular" dada por Leifer et al. (2000), la cual menciona que a las empresas maduras le es más difícil innovar en forma disruptiva (con productos nuevos).

3. Las innovaciones realizadas por las pymes consistieron mayoritariamente en "producto nuevo" 66,7\% (posiblemente una innovación radical), y en menor proporción se realizaron "productos con cambios significativos" 33,3\% (innovación incremental).

4. El mercado es principalmente "local o nacional", la muestra evidencia $91 \%$ a nivel local, y un $9 \%$ a nivel internacional.

5. El análisis de frecuencia mostro que "estudio de mercado" $87 \%$, es una de las actividades que más realizan las pymes, como parte de su proceso de innovación, seguida por "capacitación del personal" con un 80\%. 
6. Los montos más altos de inversión se evidencian en actividades de "capacitación al personal" para las pequeñas; y "adquisición de maquinarias y equipo" en las medianas empresas. También se evidenció que el gasto de innovación fue creciendo gradualmente (se solicitó información de los últimos 3 años consecutivos).

7. Las empresas en general fundamentan más sus decisiones de innovación en información proveniente de fuentes externas (proveedores, clientes y consumidores), que de fuentes científicas (organismos públicos de investigación, centro tecnológico, universidades).

8. Para financiar sus actividades de innovación, las empresas utilizaron mayoritariamente recursos propios $(74,4 \%)$, y solamente utilizaron otras fuentes de financiamiento en un tercio de los casos. Específicamente, la banca privada representó el 13\% del total.

9. Mejorar la calidad de bienes o servicios $(X=3,74$ y $S=, 966)$ fue el principal objetivo que impulsó a los empresarios a realizar el proceso de innovación, seguida de la detección de una demanda total o parcialmente insatisfecha en el mercado, o el aprovechamiento de una idea generada al interior de la firma, o por la amenaza de la competencia.

10. Acerca de los factores claves para el éxito de un proceso de innovación radical, los resultados obtenidos indican una mayor preferencia de los gerentes hacia el término "estimular la libertad de ideas" ( $X=4,05$ y $S=$ ,371); lo cual se refuerza con el análisis de la frecuencia, encontrando que el $86,4 \%$ de los gerentes consideró "importante" estimular la libertad de ideas y un $72,7 \%$ el factor de "apoyo de la gerencia y su "predisposición de romper paradigmas" ( $X=4,30$ y $S=, 462)$. Sin embargo, se considera que el factor clave para el éxito de un proceso de innovación es estimular la libertad de ideas, fundamentado en el bajo valor de la desviación estándar $(S=, 371)$, lo cual sugiere una alta coincidencia en opinión de los gerentes. 
11. Respecto a la estructura organizacional, los resultados obtenidos indican una mayor preferencia de los gerentes hacia la estructura organizacional: un equipo RRHH con personal interno $(X=0,50$ y $S=, 506)$; lo cual se refuerza con el análisis de la frecuencia, encontrando que el $50 \%$ de los gerentes considera que la estructura organizacional ideal en el proceso de innovación radical es un equipo de trabajo conformado por RRHH interno. El equipo de trabajo en promedio estuvo conformado por 4 miembros. El 27,3\% de los empresarios afirmó tener a un diseñador en el equipo de trabajo.

12. El modelo de innovación empleado fue consultado a los empresarios como una pregunta abierta, no se indujo a señalar a ningún modelo formateado, por el contrario, se solicitó que explique el modelo que había aplicado. Todas las empresas indicaron su modelo propio de innovación, que consistió en aplicar el modelo correspondiente al desarrollo de un producto, lo que se constituye para éstas en su secreto empresarial. El 9\% de las empresas indicaron que han escuchado sobre la metodología Design Thinking en un taller empresarial, pero que desconocían de los profesionales que la aplican.

Teóricamente, la puesta en marcha de la innovación según varios autores requiere de un modelo conceptual, cuyo objetivo es guiar el proceso de innovación en forma eficaz (Kelly \& Littman, 2010). El primer intento de resumir, analizar y comparar los modelos del proceso de innovación lo hace el autor Roy Rothwell (1994), quien identificó y clasificó los modelos en 5 generaciones. Posterior a él, los autores Velasco et al. (2005) detallan todos los modelos de innovación que surgieron en el período de 1983 hasta el 2004 (30 en total). Los modelos de la primera generación caracterizan al proceso como un sistema rígido con etapas lineales y la quinta, en cambio, como un proceso sistémico y en red, donde las actividades se desarrollan de manera conjunta y simultánea (en paralelo) (Rothwell, 1994, p. 22). Finalmente, el 
estudio de estos modelos ha permitido que las empresas hayan implementado e interiorizado con éxito los procesos de innovación de "Idea-a-Lanzamiento" para conceptualizar, desarrollar y comercializar nuevos productos y servicios. Comúnmente, estos procesos son conocidos como el modelo Stage-Gate que abarca las fases de generación de ideas, validar ideas, desarrollo, pruebas y lanzamiento (Cooper, 2008) (ver Figura 2).

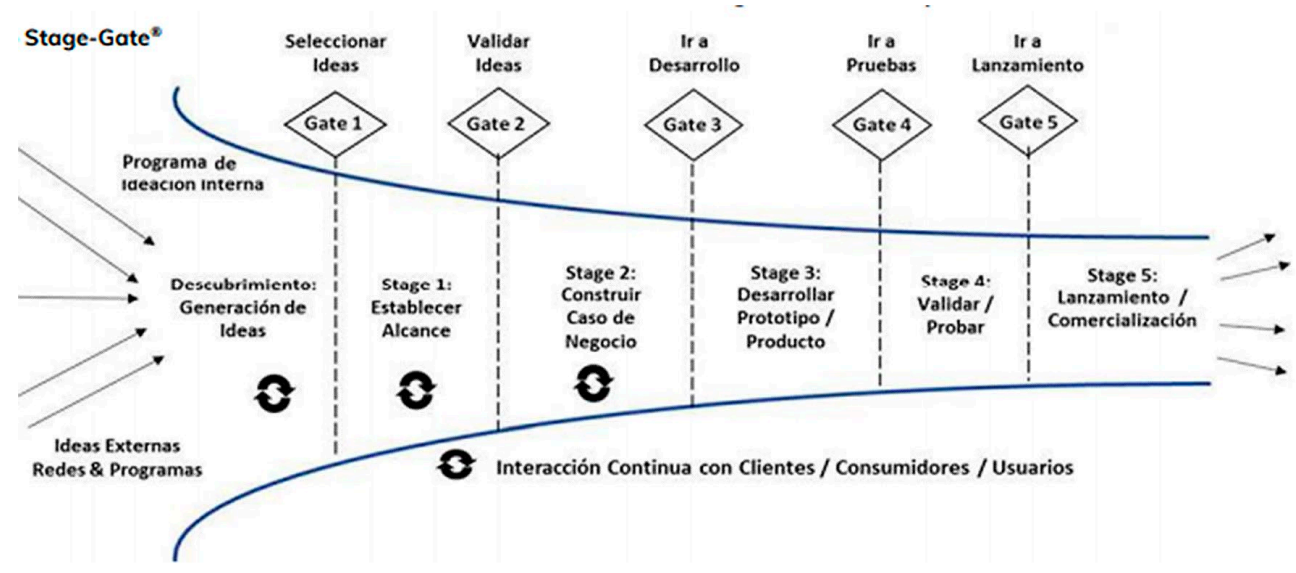




\section{Componente 3. Accionar del diseño}

En este constructo los gerentes opinaron sobre los 4 factores que estimulan y promueven el proceso de diseño en el desarrollo de la innovación: nivel de uso, fuentes creativas, herramientas de conocimiento del diseño y capacidades básicas del diseñador:

1. Nivel de uso, posicionamiento en la Design Ladder. Los empresarios identificaron las etapas que habían conformado para realizar el proceso de innovación y el rol del diseño en cada una de ellas. Estas respuestas fueron categorizadas y ubicadas en uno de los 4 escalones de Design Ladder, modelo de medición desarrollado en 2001 por el Danish Design Centre, que proporciona las directrices de cómo se usa el diseño en la empresa y, según su integración, sitúa a la entidad en uno de los cuatro escalones. La ideología es que a medida que se asciende en la escalera, se mejora el uso del diseño y adquiere mayor presencia en la empresa (ADCV, 2018).

Los resultados evidencian que el $72,72 \%$ de las empresas afirman que el diseño no tenía ningún papel en absoluto, ya que perciben el diseño estrechamente relacionado con el marketing, ante esto el rol del diseño es invisible. El 18,10\% usa al diseño a nivel estético, es decir se usa como elemento final para dar forma a los nuevos productos o servicios; el 7,08\% reconoce al diseño como un proceso, que intervino en varias etapas del proceso de innovación, el 2,10\% usaron al diseño para el desarrollo del concepto de innovación al inicio de este (ver Figura 3). 


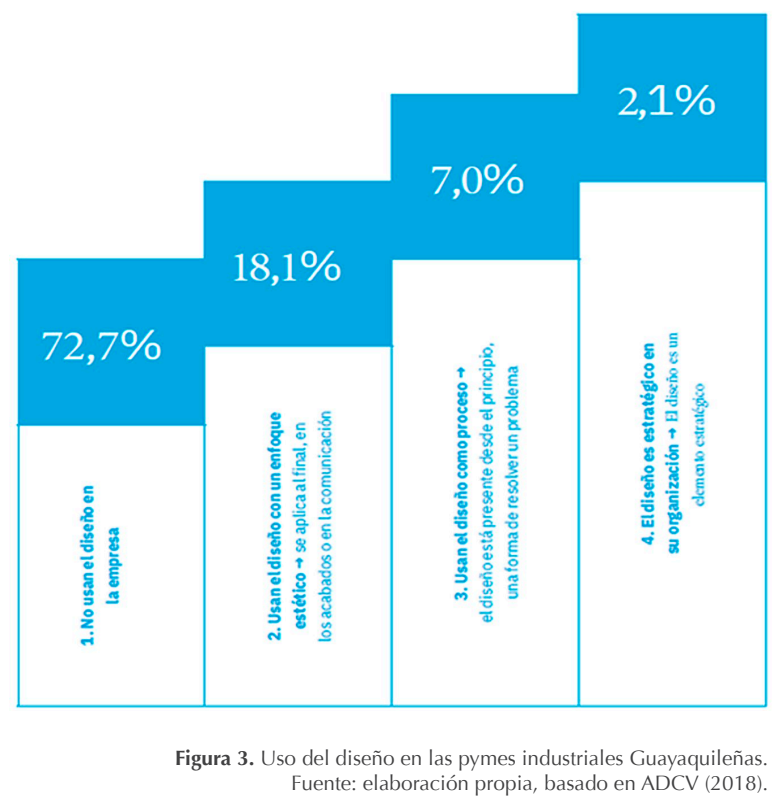

2. Los resultados también invitan a reflexionar sobre el porcentaje mayoritario que afirma no emplear diseño; la observación cualitativa permitió identificar un desconocimiento absoluto sobre el potencial del diseño, pues se ve asociado más con el concepto de marketing. Lo que dejar ver un amplio camino por recorrer de los profesionales de diseño o de la academia con respecto a comunicar sobre el alcance y el sentido de su práctica y de sus resultados.

3. Se evidenció que las fuentes creativas provienen principalmente del entorno, los resultados indican una mayor preferencia hacia la fuente interna: diálogo con la alta gerencia $(X=3,65$ y $S=1,089)$ y como fuente externa las regulaciones económicas $(X=3,88$ y $\mathrm{S}=, 879)$. El análisis de 
frecuencia, por su parte, evidenció que el 73,2\% de los gerentes consideró importante las "políticas públicas del territorio" y "las regulaciones económicas".

4. Con respecto a las herramientas, se destacan a gestionar la complejidad $(X=3,58$ y $S=, 932)$, trabajar en contexto $(X=4,08$ y $S=, 932)$ y aplicar empatía al contexto del problema.

5. Las capacidades básicas de los diseñadores, se identificaron con los resultados obtenidos, existe una mayor preferencia de los gerentes hacia los términos: trabajo en equipo $(X=4,75$ y $S=, 452)$ y visión sistemática $(X=4,67$ y $S=, 492)$; lo cual se refuerza con el análisis de frecuencia, encontrando que el $75 \%$ de los gerentes consideró "muy importante" la habilidad de trabajo en equipo y el $66,7 \%$ considero "muy importante" la visión sistemática, sin embargo, se considera que el término que define la habilidad del diseñador en la generación de innovación radical es el trabajo en equipo, fundamentado en el bajo valor de la desviación estándar $(\mathrm{S}=, 452)$.

\section{Conclusiones}

La relación diseño e innovación radical, desde la óptica documental, cuenta con una vasta bibliografía procedente de territorios europeos. Se destaca el papel protagónico del diseñador en la etapa de "generación de ideas" mediante la investigación de diseño, la que proporciona información sobre las necesidades de los clientes mediante el uso de la investigación etnográfica en un contexto sociocultural, información que se triangula con la investigación de mercado y la tecnológica. Hoy en día los investigadores denotan que el estudio de las necesidades desde un punto de vista cualitativo, junto con la capacidad de anticipar los gustos e intereses de un grupo de consumidores 
a partir de la detección temprana de indicios de cambio en sus patrones de consumo y los valores que los inspiran, es la pieza angular en la innovación. Y es precisamente este el rol que cumple el diseñador en diversas etapas del proceso de innovación, dadas sus habilidades y metodologías vinculadas a la observación, la comprensión del mercado, la generación de ideas, su conceptualización y visualización de un futuro imaginario (Carrió, 2006).

La relación vista en un territorio particular de Latinoamérica evidenció que el 9\% de las pymes consultadas integró al diseño en el proceso de innovación, lo que conduce a la siguiente pregunta ¿cómo innovaron sin diseño las empresas restantes?, esta pregunta tiene muchas respuestas. a) En la generación de ideas inclinaron su balanza hacia datos cuantitativos para la búsqueda de nuevas oportunidades, empleando para ello solo personal técnico especializado. b) El rol del diseñador es invisible ante el empresario, este se comercializa como una actividad del marketing; la empresa contrata al marketing para estudios de mercado, para creación de marca, empaques y campañas publicitarias. Los 66 empresarios consultados afirmaron tener el área de marketing dentro de su proceso de innovación. c) Las empresas que afirmaron usar diseño en el proceso de innovación tuvieron que contratar diseñadores extranjeros, pues afirmaron no haber encontrado diseñadores locales con experiencia en innovación.

Respecto a las habilidades que deben poseer los diseñadores para integrarse al ámbito estratégico en el proceso de innovación, los empresarios (que usaron diseño) coinciden con la revisión bibliográfica en tres habilidades fundamentales (visión sistémica, creatividad y trabajo en equipo). Enfatizan la capacidad de visualizar la problemática en todas sus dimensiones (visión sistémica), donde confluyen aspectos del macro y microentorno que afectan a la empresa. El manejo de herramientas o metodologías administrativas que le permitan hablar en términos de modelo de negocio y visualizar todo el entorno del producto, en su escenario material, de transformación, de comunicación y consumo. 
Creativos, despabilados y proactivos son los adjetivos con que califican los empresarios al diseñador. Que no se encierre en su mundo creativo, sino que participe activamente de las discusiones en las reuniones de innovación, que no tenga temor al expresar sus ideas y que las defienda con fortalezas. Que sea un interlocutor que escucha, analiza, interpreta y propone con expertos de otras áreas el entendimiento de un problema y llegue a múltiples soluciones relevantes, coherentes y pertinentes. Es decir que trabaje en equipo.

La tarea pendiente es integrar y visibilizar el diseño en el proceso de innovación en el territorio estudiado. La visión sistémica de esta investigación identifica varios agentes. Por ejemplo, la academia puede evaluar los resultados y desarrollar las iniciativas que permitan superar esta brecha de conocimiento; pero no solo tener un profesional capacitado ayuda en el proceso, también es necesario trabajar con el empresario, se requiere capacitación sobre la gestión innovadora de la empresa y una mejor comprensión de cómo y dónde el diseño aportará al proceso de innovación; dado que la exploración de escenarios más innovadores requiere una alineación estratégica constante y un compromiso real de la alta gerencia. Finalmente, el Estado también debe contribuir con políticas públicas que permitan mejorar la comprensión del impacto del diseño en la innovación.

\section{Referencias}

ADCV. (2018). La economía del diseño en la comunitat Valenciana. Agència Valenciana de la Innovació (Generalitat Valenciana). https://www.adcv. com/proyecto/economia-disseny/

Battistella, C., Biotto, G., \& De Toni, A. F. (2012). From design driven innovation to meaning strategy. Management Decision, 50(4), 718-743. http://dx.doi. org/10.1108/00251741211220390 
BID. (2016). Innovación y productividad en las empresas en América Latina y el Caribe: el motor del desarrollo económico (Matteo Gra). New York. https:// publications.iadb.org/publications/spanish/document/Innovaci\%C3\%B3ny-productividad-en-las-empresas-en-Am\%C3\%A9rica-Latina-y-el-CaribeEl-motor-del-desarrollo-econ\%C3\%B3mico-(Resumen).pdf

Borja De Mozota, B. (2006). El diseño de la innovación, dos retos para la profesión del diseño. Innovación y Diseño, 23, 132-146. https://www. raco.cat/index.php/Temes/article/view/51784/104097

Botella, C., \& Suárez, I. (2012). Innovación para el desarrollo en América Latina: Una aproximación desde la cooperación internacional. Fundacion Carolina, 78, 20-25. https://www.fundacioncarolina.es/wp-content/ uploads/2014/08/AI78.pdf

Cámara de Industrias de Guayaquil. (agosto de 2019). La estructura de abastecimiento en la manufactura ecuatoriana. INDUSTRIAS, 11-15. https://issuu.com/industrias/docs/revista_industria_agosto_2019

Cárdenas, F. (2015). El sector alimenticio del Ecuador se toma el pulso. Gestión, 251, 24-26. https://revistagestion.ec/sites/default/files/import/ legacy_pdfs/251_003.pdf

Castañeda, M., Cabrera, A., Navarro, Y., \& Wietse, V. (2010). Procesamiento de datos y analisis estadísticos utilizando SPSS. EDIPUCRS. http://www.pucrs. br/orgaos/edipucrs

Chesbrough, H. (2003). Open Innovation: The New Imperative for Creating And Profiting from Technology. Harvard Business School.

Carranco, R. (2017). La aportación de las pequeñas y medianas empresas (pymes) en la economía ecuatoriana. Revista Ciencia Administrativa (2), 146-157. https://www.uv.mx/iiesca/files/2018/03/14CA201702.pdf 
Carrió, M. (2006). Espacios comunes del diseño y la innovación. Innovacion y Diseño, 23, 147-155. https://www.raco.cat/index.php/Temes/article/ view/51785/104113

CEPAL. (20 de 07 de 2013). Como mejorar la competitividad de la Pymes en la Unión Europea y América Latina y el Caribe: propuestas de política del sector privado. https:/www.cepal.org/es/publicaciones/3094-comomejorar-la-competitividad-pymes-la-union-europea-america-latina-caribe

Chandy, R. K., \& Tellis, G. J. (2000). The Incumbent's Curse? Incumbency, Size, and Radical Product Innovation. Journal of Marketing, 64(2), 1-17. https://doi.org/10.1509\%2Fjmkg.64.3.1.18033

Cooper, R. (2008). Maximizing Productivity in Product Innovation. Research Technology Management. https://www.researchgate.net/publication/ 263080371_Maximizing_Productivity_in_Product_Innovation.

Cotec. (2009). Productividad, competitividad e innovación en España: comparación internacional por sectores. Fundación Cotec. https://ocw.bib. upct.es/pluginfile.php/6387/mod_resource/content/1/Estudio_2009.pdf

Cruickshank, L. (2010). The Innovation Dimension: Designing in a Broader Context. Design Issues, 26(2), 17-26. https://www.jstor.org/stable/20749939

Damanpour, F. (1991). Organizational innovation: A meta-analysis of effects of determinants and moderators. Academy of Management Journal, 34(3), 550-590. https://doi.org/10.5465/256406

Dell'Era, C., \& Verganti, R. (2007). Strategies of innovation and imitation of product languages. Journal of Product Innovation Management, 24(6), 580-599. https://doi.org/10.1111/j.1540-5885.2007.00273.x

Doing Business. (21 de 07 de 2017). Doing Business. Obtenido de http://espanol.doingbusiness.org/ 
Guaipatín, C., \& Schwartz, L. (2014). Ecuador, Análisis del Sistema Nacional de Innovación. BID, Division de Competitividad e Innovación.

https://publications.iadb.org/es/ecuador-analisis-del-sistema-nacional-deinnovacion-hacia-la-consolidacion-de-una-cultura

Hobday, M., Boddington, A., \& Grantham, A. (2012). Policies for design and policies for innovation: Contrasting perspectives and remaining challenges. Technovation, 32(5), 272-281. http://dx.doi.org/10.1016/j. technovation.2011.12.002

INEC. (2015). Principales indicadores de actividades de ciencia, tecnología e innovación (ACTI): 2002-2014. http://www.ecuadorencifras.gob.ec/ documentos

Jones, M. \& Samalionis, F. (2008). From Small Ideas to Radical Service Innovation. Design Management Review, Winter, 20-26. https://doi. org/10.1111/j.1948-7169.2008.tb00102.x

Kelly, T., \& Littman, J. (2010). Las diez caras de la innovación. Barcelona: España.

Leifer, R., McDermott, C., Colarelli, G., Peters, L., Rice, M. \& Veryzer, R. (2000). Radical Innovation: How Mature Companies Can Outsmart Upstarts. Rensselaer Polytechnic Institute.

Lemarchand, G. a. (2010). Sistemas nacionales de ciencia, tecnología e innovación en América Latina y el Caribe. UNESCO, 329.https://www.epn. edu.ec/wp-content/uploads/2017/03/sistem-nacion-cyt.pdf

Namakforoosh, M. (2005). Metodología de la investigación. México: Limusa. 
Norman, D. \& Verganti, R. (2014). Incremental and radical Innovation: Design rearch vs technology and Meaning Change. Designlssues, 30(1), 79-96. http://www.verganti.com/wp-content/uploads/2017/01/Norman Verganti.pdf

Noble, C. (2011). On Elevating Strategic Design Research. Journal of Innovation Managment, 389-393. https://doi.org/10.1111/j.1540-5885.2011.00808.x

OCDE \& EUROSTAT. (2005). Manual de Oslo, Guia para la recogida e interpretación de datos sobre innovación. Tagsa. http://www.oecd.org/ centrodemexico/laocde/

Property Rights Alliance. (20 de 07 de 2017). INTERNATIONAL. Obtenido de https://internationalpropertyrightsindex.org/

RICYT. (22 de 08 de 2017). Red de Indicadores de Ciencia y Tecnología -Iberoamericana e Interamericana. Obtenido de http://www.ricyt.org/

Rothwell, R. (1994). Towards the Fifth-generation Innovation Process. International Marketing Review, 11(1), 7-31. https://doi. org/10.1108/02651339410057491

Slater, S. F., Mohr, J. J. \& Sengupta, S. (2014). Radical product innovation capability: Literature review, synthesis, and illustrative research propositions. Journal of Product Innovation Management, 31(3), 552-566. http://doi. org/10.1111/jpim.12113

Tether, B. (2005). The Role of Design in Business Performance. Design, 1-26. http://www.bis.gov.uk/files/file14796.pdf

Velasco, E., Zamanillo, I., \& Gurutze, M. (2005). Evolución de los modelos sobre el proceso de innovación: Desde el modelo líneal hasta los sistemas de innovación. Decisiones Organizativas, 1-15. http://doi.org/10.1111/ aman. 12336 
Yannou, B., Jankovic, M., \& Leroy, Y. (2011). Empirical verifications of some radical innovation design principles onto the quality of innovative designs. In International Conference on Ingineering Design, ICED 11. https://www. designsociety.org/publication/30420/EMPIRICAL+VERIFICATIONS+OF+S OME+RADICAL+INNOVATION+DESIGN+PRINCIPLES+ONTO+THE+Q UALITY+OF+INNOVATIVE+DESIGNS

Zúñiga, P. (2010). La necesidad de innovar. El camino hacia el progreso de América Latina y el Caribe. Banco Interamericano de Desarrollo. https://publications.iadb.org/es/la-necesidad-de-innovar-el-camino-haciael-progreso-de-america-latina-y-el-caribe

Zurlo, F. \& Cautela, C. (2014). Design Strategies in Different. Designlssues, 30(1), 19-35. https://www.jstor.org/stable/24267023

Cómo citar: Matovelle, R., Lecuona, M. \& Hernandis, B. (2021). Diseño e innovación radical desde una visión sistémica: el caso de las pymes industriales de Guayaquil-Ecuador. Revista KEPES, 18(23), 49-74. https://doi.org/10.17151/kepes.2021.18.23.3 